\title{
Reconstruction of bony facial contour deficiencies with polymethylmethacrylate implants: case report
}

\author{
Ruy C. C. ABDO FILHO', Thais M. OLIVEIRA², Natalino LOURENÇO NETO ${ }^{3}$, Carla GURGEL', Ruy C.C. ABDO ${ }^{4}$
}

\begin{abstract}
1- DDS, Assistant Professor, Department of Oral Surgery, School of Dentistry, University of Alfenas, Alfenas, MG, Brazil.
2- DDS, PhD, Assistant Professor, Department of Pediatric Dentistry, Orthodontics and Community Health, Bauru School of Dentistry, University of São Paulo, Bauru, SP, Brazil.

3- DDS, MSc, PhD student, Department of Pediatric Dentistry, Orthodontics and Community Health, Bauru School of Dentistry, University of São Paulo, Bauru, SP, Brazil.

4- DDS, PhD, Full Professor, Department of Pediatric Dentistry, Orthodontics and Community Health, Bauru School of Dentistry, University of São Paulo, Bauru, SP, Brazil.
\end{abstract}

Corresponding address: Prof. Dr. Ruy Cesar Camargo Abdo - Faculdade de Odontologia de Bauru - USP - Departamento de Odontopediatria, Ortodontia e Saúde Coletiva - Disciplina de Odontopediatria - Alameda Dr. Octávio Pinheiro Brisolla, 9-75 - Bauru, São Paulo - $17012-901$ - Brasil - Phone: 551432358218 e-mail: marchini@usp.br

Received: March 25, 2010 - Modification: May 30, 2010 - Accepted: October 26, 2010

\section{ABSTRACT}

\begin{abstract}
Eacial trauma can be considered one of the most serious aggressions found in the medical centers due to the emotional consequences and the possibility of deformity. In craniofacial surgery, the use of autologous bone is still the first choice for reconstructing bony defects or irregularities. When there is a shortage of donor bone or a patient refuses an intracranial operation, alloplastic materials such as polymethylmethacrylate (PMMA) can be used. The PMMA prosthesis can be pre-fabricated, bringing advantages such as reduction of surgical time, easy technical handling and good esthetic results. This paper describes the procedures for rehabilitating a patient with PMMA implants in the region of the face, recovering the facial contours and esthetics of the patient.
\end{abstract}

Key words: Polymethylmethacrylate. Craniofacial abnormalities. Prostheses and implants. Facial injuries.

\section{INTRODUCTION}

Facial trauma can be considered one of the most serious aggressions found in the medical centers due to the emotional consequences and the possibility of deformity. This is an injury that requires a multidisciplinary approach involving several specialties such as ophthalmology, plastic surgery, maxillofacial surgery and neurosurgery. Posttraumatic facial restoration requires the combination of both esthetic and reconstructive principles, and both had great progress in the last decade ${ }^{14}$.

The large range of techniques and rehabilitation procedures in this field includes the transplantation of autogenous or heterogenous hard tissue and the implantation of alloplastic materials that can be modeled during or before the surgery ${ }^{8}$. In craniofacial surgery, the use of autogenous bone is still the first choice for reconstructing bony defects or irregularities, but sometimes the patient refuses another surgery to obtain the transplantation, so alloplastic materials are required for these cases $^{3}$.

Nowadays the most used alloplastic material by surgeons for this rehabilitation is the polymethylmethacrylate (PMMA), which is an acrylic-based resin, biocompatible, nondegradable material. PMMA prostheses can be pre-fabricated, thus bringing advantages such as reduction of surgical time, easy technical handling and good esthetic results ${ }^{1-3,6,7,10}$.

The aim of this work was to describe the procedures for rehabilitating a patient with PMMA implants in the region of the face, recovering the facial contours and esthetics of the patient. 


\section{CASE REPORT}

A 20-year-old male patient was referred to our clinic in 2008 with a facial defect in the zygomatic area as a result of a trauma occurred 7 years before. According to the patient's report, he went to the local emergency hospital immediately after the trauma and was medicated, but no treatment was performed at that moment. However, after the regression of the swelling, he noted a depression on his face in the region of the trauma and since then he had looked for treatment to this condition.

Clinical and radiographic examination showed that the facial trauma had caused a fracture with zygomatic bone depression. After a period of 7 years, a bone consolidation in the inadequate position had occurred and a facial depression in the area had compromised facial esthetics (Figure 1A, $B, C$ and D). After the preoperative discussion, the treatment of choice was the use of PMMA for filling the facial defect.

Initially an impression of the patient's face was made using alginate material. The impression was cast with dental stone (Figure $2 \mathrm{~A}$ and $\mathrm{B}$ ). On the cast, the facial prosthesis was sculpted in wax. After that, the wax piece was embedded in a dental flask replaced with PMMA by an exothermic reaction according to the manufacturer's instructions. Three pieces with same format and different sizes were prepared and sterilized in ethylene oxide before surgery. Several different framework designs, shapes, levels of stiffness, and thicknesses were tried on the cast, and then evaluated on the patient. The pieces were tried on the patient to obtain the appropriate shape for the prosthesis (Figure 3A, $B$ and $\mathrm{C})$.

Preoperative exams were carried out and the surgical procedure was performed under general anesthesia. Intraoral access to the zygomatic area was made through an incision in the superior left labial vestibule (Figure 4A, B and C). After the dissection of the mucoperiosteal flap, the infraorbital nerve foramen was identified and the defective area was accessed. It was observed a little communication between the anterior bony wall and the maxillary sinus (Figure 4D). In order to prevent any possible contamination, a fibrin sponge was placed in this area (Figure 4E).

During surgery, the appropriate size was chosen and inserted in the defective contour. Minimal refining was necessary and recontoured by trimming was done with an acrylic bur (Figure $4 \mathrm{~F})$. The prosthesis was fixed with three titanium screws $(2.0 \times 12 \mathrm{~mm}$ ) (Figure $4 \mathrm{G})$. Finally, the mucoperiosteal flaps were repositioned and closed with Vicryl sutures (polyglactin 910) (Figure 4H).

Right after surgery, the patient was instructed to adopt a pasty diet and to avoid toothbrushing
A

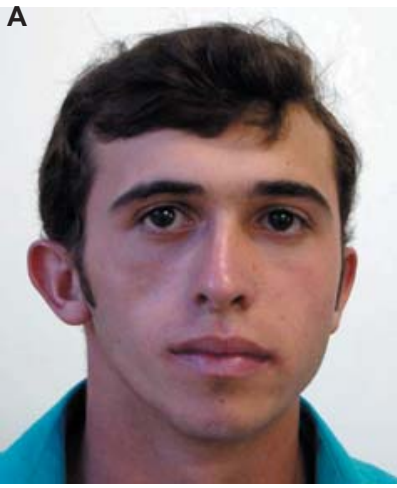

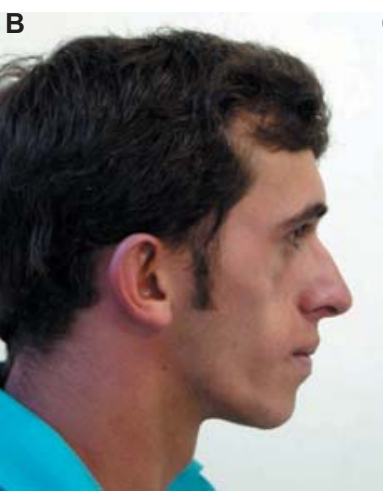

C
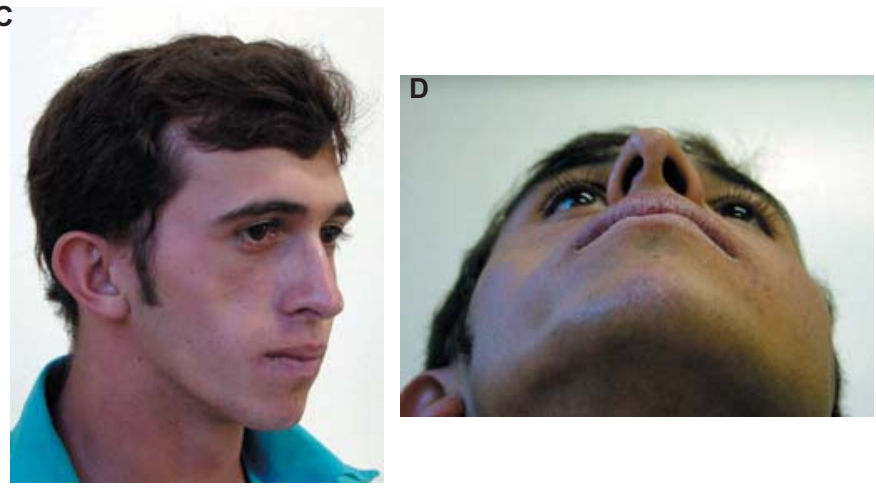

Figure 1A-D- Facial depression compromised the facial esthetics (patient signed informed consent authorizing the publication of these pictures)
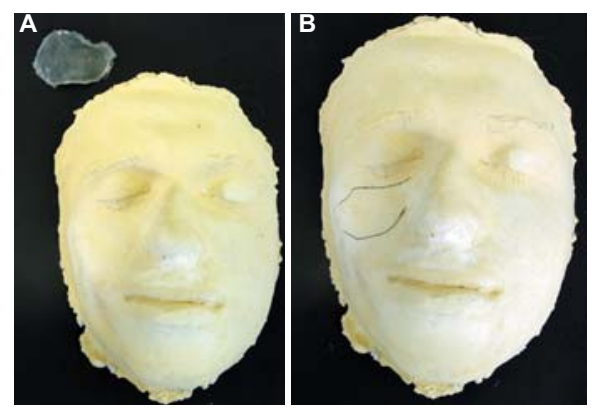

Figure 2A-B- Impression of the patient's face with alginate
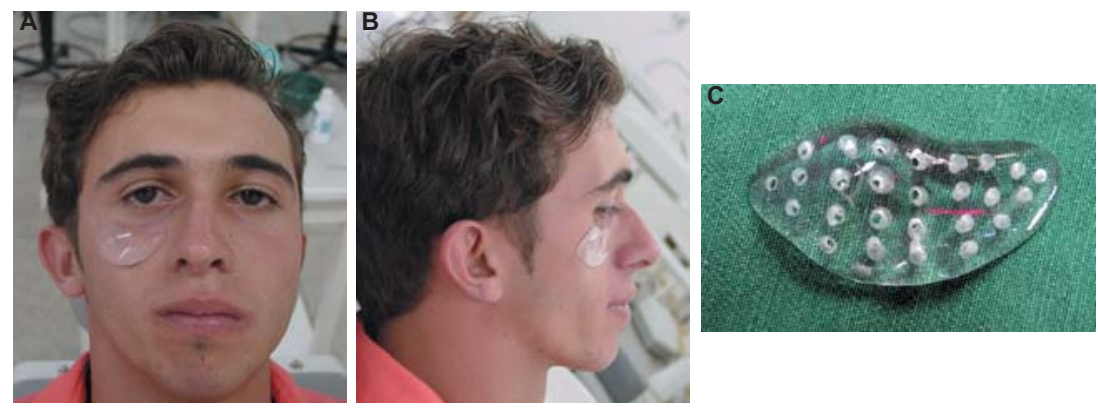

Figure 3A-C- The facial prosthesis was sculpted. The appropriate shape was obtained (patient signed informed consent authorizing the publication of these pictures) 

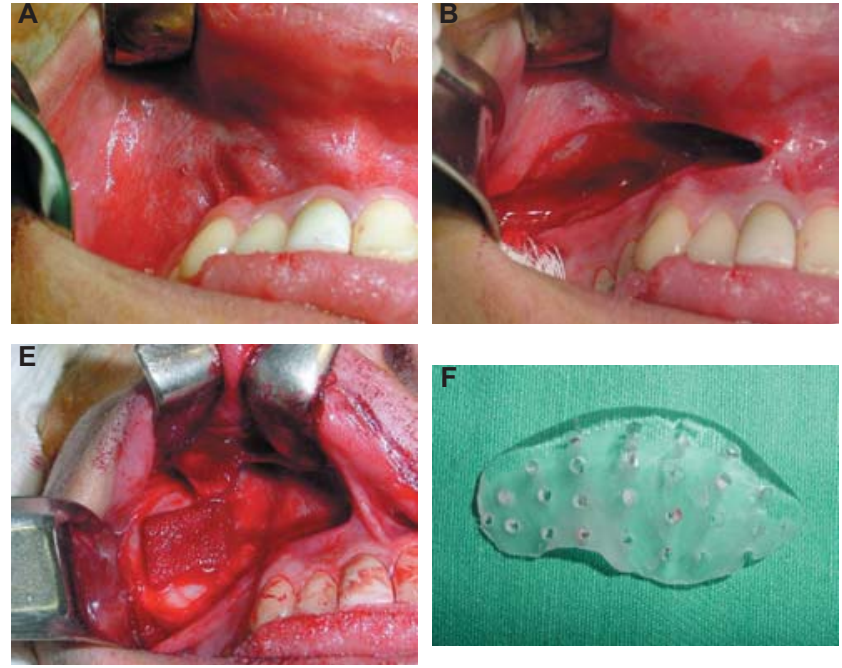
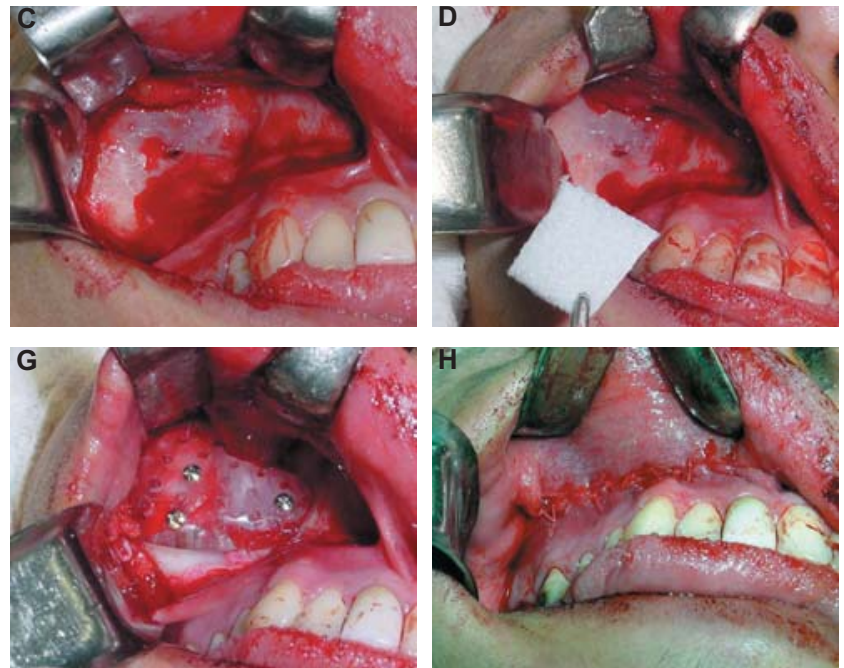

Figure 4- Panel of intraoperative photographs. A: Access to the zygomatic area. B-C: Incision in the superior left labial vestibule. D-E: Fibrin sponge placed in this area. F: Prosthesis recontoured by trimming it with an acrylic bur. G: Prosthesis fixed with three titanium screws. H: Mucoperiosteal flaps repositioned and closed with Vicryl sutures.
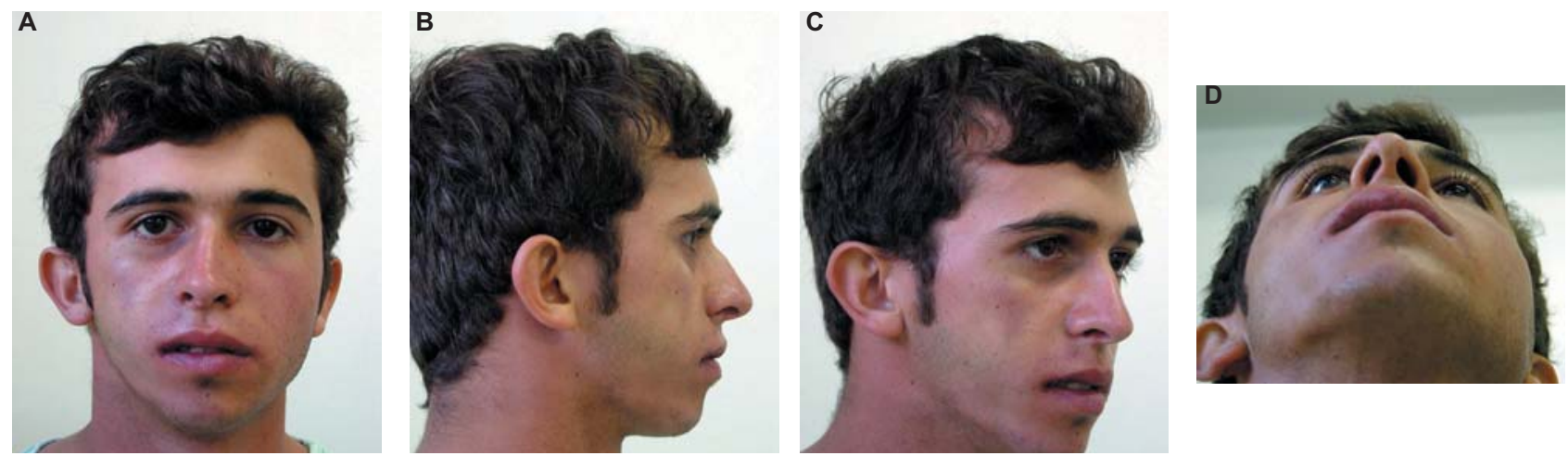

Figure 5A-D-Evaluation at 20 days postsurgery (patient signed informed consent authorizing the publication of these pictures)
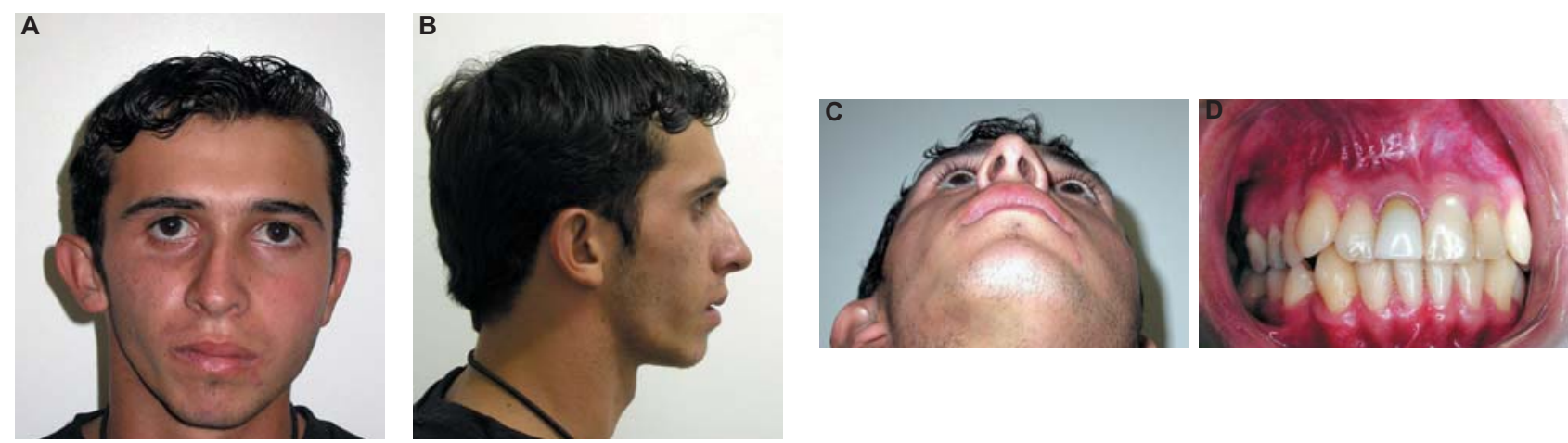

Figure 6A-D- Follow-up of 36 months (patient signed informed consent authorizing the publication of these pictures)

in the operated side for 7 days. Topical application of $0.12 \%$ chlorhexidine gluconate gel with a cotton bud in the surgical area once a day was prescribed. Also, amoxicillin $(500 \mathrm{mg})$ was administered for 7 days and dipyrone sodium (500 $\mathrm{mg}$ ) for 3 days.

Evaluation at 20 days postsurgery showed that the patient healed well with an acceptable cosmetic outcome and without any major complications (Figure 5A, B, C and D). The patient returned after 1,3 and 6 months, and every year thereafter. At
36 months of follow up, the patient presented good clinical response and was discharged (Figure 6A, $B, C$ and $D)$.

\section{DISCUSSION}

Facial trauma can affect either the bony contour, or the bony continuity, or both causing bone defects. Depending on the trauma, the quality, the site and dimension of the defect, all fields 
of cranio-maxillofacial surgery are involved 7,8 . Numerous materials may be used to reconstruct the facial trauma including autologous bone and different types synthetic resins. It is not uncommon for postcraniofacial trauma patients to require augmentation of the depressed craniofacial skeleton. The use of autogenous bone grafts appears to have been disappointing due to unpredictable resorption and possible donor-site morbidity ${ }^{4}$. However, in craniofacial surgery, the use of autologous bone is still the first choice for reconstructing bony defects or irregularities. When there is a shortage of donor bone or a patient refuses an intracranial operation, alloplastic materials such as PMMA can be used ${ }^{3}$. Although the ideal implant material has not yet been found, numerous possibilities are available for surgeons, which provide attractive alternatives to autogenous bone graft in the appropriate clinical settings. The ease and safety of use of the alloplastic materials enables them to be used effectively and play an important role in reducing operating times ${ }^{7,10}$.

Various types of replacement materials have been used. With a complex range of possibilities, the synthetic material should be biocompatible, inert, non-thermal conducting, radio-transparent, non-magnetic, lightweight, rigid, simple to prepare, easily applicable and inexpensive e $^{5,711,13}$.

Today, the most commonly materials used fall into four groups: autografts, allografts, xenografts and alloplastic materials $s^{6,7,10}$. As a result, alloplastic materials are chosen more often. One of the main advantages of using alloplastic materials is that it is possible to prefabricate the prosthesis preoperatively, thus, reducing the operative time and complexity, and improving the final result ${ }^{6}$. Some authors believe that PMMA is one of the inert materials that best fulfills most of these requirements ${ }^{5,6,11,13}$. It is commonly used because of its remarkable plasticity and long-term stability. Moreover, it does not interfere with radiographs or electroencephalograms or radiotherapy. PMMA is also inexpensive and easily available. Overtime, PMMA has been shown to be well tolerated without presenting biological side effects like foreign-body reactions $5,6,11,13$.

PMMA is composed by fine particles of prepolymerized and mixed with methylmethacrylate (MMA). The polymerization process is triggered by the reaction between the benzoyl peroxide in the polymer powder with $\mathrm{N}, \mathrm{N}$-dimethyl-p-toluidine. A radiopacifier, such as barium sulfide or zirconium dioxide, is also added to the powder component. MMA polymerization is highly exothermic and its temperature can exceed $80^{\circ} \mathrm{C}$. PMMA is actually well tolerated and bone tissue may be formed at its surface. After the polymerization, usually 15 min, about $3-5 \%$ of the monomer remains, and decreases to $1-2 \%$ with time. The monomer toxicity should disappear within $4 \mathrm{~h}^{12}$. Lu, et al. ${ }^{12}$ (2002) did not found any toxicity of PMMA after 48 and 78 months of implantation. In addition, the authors reported no MMA monomer released fragments after implantation.

Some disadvantages should be mentioned. PMMA is not incorporated into or vascularized by adjacent bone, it may be subject to infection and, in cases of cold polymerization, residual monomer may be toxic ${ }^{6}$. In the present case PMMA was used for reconstruction of bony facial contour deficiencies after facial trauma. However, prefabricating PMMA, as done in this case report, has several advantages. These include complete polymerization resulting in no permeability to body fluids, shortening of operative time, and ensuring to the achievement of improved physical properties, such as compressive, impact and shear strength. The technology involved is simple and easily accessible ${ }^{1}$. The authors used simple impression material commonly employed in dentistry to obtain the impression of the shape of the bone defect. This technique, apart from being affordable, also ensures shorter operative time and good esthetic result.

Allergic reactions to PMMA are rare. When they occur, it is a reaction against one of the components of the PMMA such as N, N-dimethyl-p-toluidine, which is used as an accelerator, or monomer MMA. A small percentage of the population is allergic to MMA, and it can cause the burning mouth syndrome, stomatitis, or chronic urticaria in patients wearing a dental prosthesis ${ }^{3,9}$. The patient of this case report did not present any discomfort; PMMA was well accepted and showed good response after 3 years of follow-up.

A reconstructive surgeon attempts to bring the face back to the preinjury condition. An esthetic surgeon attempts to improve upon the existing normal. The maxillofacial surgeons should endeavor to do both. The progress in art and science allows taking the facial reconstruction to facial restoration. The approach to the trauma patient should begin with observation. Recent photographs are helpful and the surgeon can often make an accurate diagnosis based on observation and physical examination ${ }^{14}$.

The experience with the use of pre-fabricated PMMA prosthesis showed remarkable advantages, as it was precise when compared with another case and inexpensive. The PMMA prosthesis is an attractive alternative to other high-tech procedures. 


\section{REFERENCES}

1- Abdulai A, Iddrissu M, Dakurah T. Cranioplasty using polymethyl methacrylate implant constructed from an alginate impression and wax elimination technique. Ghana Med J. 2006;40(1):18-21. 2- Al Ruhaimi KA. Bone graft substitutes: a comparative qualitative histologic review of current osteoconductive grafting materials. Int J Oral Maxillofac Implants. 2001;16(1):105-14.

3- Bruens ML, Pieterman H, De Wijn JR, Vaandrager JM. Porous polymethylmethacrylate as bone substitute in the craniofacial area. J Craniofac Surg. 2003;14(1):63-8.

4- Chen TM, Wang HJ, Chen SL, Lin FH. Reconstruction of posttraumatic frontal-bone depression using hydroxyapatite cement. Ann Plast Surg. 2004;52(3):303-8.

5- Cheng AC, Wee AG. Reconstruction of cranial bone defects using alloplastic implants produced from a stereolithographicallygenerated cranial model. Ann Acad Med Singapore. 1999;28(5):6926.

6- Chiarini L, Figurelli S, Pollastri G, Torcia E, Ferrari F, Albanese $M$, et al. Cranioplasty using acrylic material: a new technical procedure. J Craniomaxillofac Surg. 2004;32(1):5-9.
7- Cho YR, Gosain AK. Biomaterials in craniofacial reconstruction. Clin Plast Surg. 2004;31(3):377-85.

8- Eufinger $\mathrm{H}$, Wehmöller M, Harders A, Heuser L. Prefabricated prostheses for the reconstruction of skull defects. Int J Oral Maxillofac Surg. 1995;24:104-10.

9- Fisher AA. Paresthesia of the fingers accompanying dermatitis due to methylmethacrylate bone cement. Contact Dermatitis. 1979;5:56-7.

10- Gosain AK, Persing JA. Biomaterials in the face: benefits and risks. J Craniofac Surg. 1999;10:404-14.

11- Lara WC, Schweitzer J, Lewis RP, Odum BC, Edlich RF, Gramper TJ. Technical considerations in the use of polymethylmethacrylate in cranioplasty. J Long Term Eff Med Implants. 1998;8:43-53.

12- Lu JX, Huang ZW, Tropiano P, Clouet D'Orval B, Remusat M, Dejou J, et al. Human biological reactions at the interface between bone tissue and polymethylmethacrylate cement. J Mater Sci Mater Med. 2002;13(8):803-9.

13- Moreira-Gonzalez A, Jackson IT, Miyawaki T, Barakat K, DiNick V. Clinical outcome in cranioplasty: critical review in long-term follow-up. J. Craniofac. Surg. 2003;14(5):144-53.

14- Wexler A. Posttraumatic facial restoration: a philosophy of care. J Craniofac Surg. 2009;20(1):12-4. 